\title{
Negative correlation between terminal velocity and VHF radar reflectivity: observation and plausible explanation
}

\author{
C.-L. Su, Y.-H. Chu, and I.-Y. Lo \\ Institute of Space Science, National Central University, Chung-Li, R.O.C. Taiwan \\ Received: 7 November 2007 - Revised: 3 March 2009 - Accepted: 3 March 2009 - Published: 6 April 2009
}

\begin{abstract}
The correlation between precipitation backscatter $P$ (or radar reflectivity $Z$ ) and reflectivity-weighted terminal velocity $V_{t}$ has long been thought to be positive. Namely, the larger the magnitude of the terminal velocity is, the stronger the radar reflectivity will be, and vice versa. However, we will show in this article the observational evidences of negative $V_{t}-P$ correlation made with the Chung-Li VHF radar. It is found that the negative $V_{t}-P$ correlation can occur in the regions from close to ground to well above the melting layer. In addition, there is a strong tendency for the negative $V_{t}-P$ correlation to occur around the bright band region. In light of the fact that the conventional model of single drop size distribution cannot explain this negative correlation, it is proposed that the drop size distribution responsible for the negative $V_{t}-Z$ correlation is composed of two Gamma drop size distributions with respective mean terminal velocities and radar reflectivities. The precipitation particles of these two distributions are assumed to dynamically interact in the way that the total numbers of the precipitation particles of the two Gamma distributions are varied and their reflectivities are also changed accordingly. Theoretical analysis shows that the key factor determining the sign of the $V_{t}-Z$ correlation is the ratio of the difference between relative changes in the reflectivities of the two Gamma drop size distributions to the change in the total reflectivity. The $V_{t}-Z$ correlation is negative (or positive) if the ratio is positive (or negative). From these results, it follows that the $V_{t}-Z$ correlation could be considered to be the result of the redistribution of the radar reflectivies of the two Gamma drop size distributions caused by the interaction of the precipitation particles between them. Different interaction processes of the precipitation particles, such as break-up and coalescence, could give rise to the same $V_{t}-Z$ correlation, depending on the net change in the total
\end{abstract}

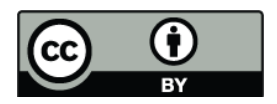

Correspondence to: Y.-H. Chu (yhchu@jupiter.ss.ncu.edu.tw) reflectivity. In addition, the results also show that the same interaction process might give opposite $V_{t}-Z$ correlations. Therefore, great caution is advised when the $V_{t}-Z$ correlation is employed to interpret the precipitation process.

Keywords. Meteorology and atmospheric dynamics (Precipitation; Instruments and techniques) - Radio science (Remote sensing)

\section{Introduction}

Terminal velocity and reflectivity of hydrometeor are two basic parameters characterized the behavior of the precipitation. With its capability of simultaneously measuring Doppler velocities of atmospheric refractivity fluctuations and precipitation particles $V_{D}$, the VHF Doppler radar (or wind profiler) can directly obtain the information on the terminal velocity and the intensity of the precipitation from the radar returns from the precipitating environment (Fukao et al., 1985; Larsen and Rottger, 1987; Chu and Lin, 1994). Note that only reflectivity-weighted terminal velocity $V_{t}$ and vertical air velocity $V_{a}$ can be observed by a VHF radar. Because the Doppler measurement of the precipitation echoes is the combination of $V_{t}$ and $V_{a}$, true $V_{t}$ can be obtained by removing $V_{a}$ from $V_{D}$. The relations between $V_{t}$ and radar reflectivity $Z$ (in unit $\mathrm{m}^{-3} \mathrm{~mm}^{-6}$ ) have been extensively used in radar meteorology to correct measurements of mean Doppler velocity for the presence of vertical winds (Battan, 1973; Ulbrich and Chilson, 1994). With VHF/UHF radars, Chilson et al. (1993) analyzed the relationship between $V_{D}$ and $Z$ associated with a thunderstorm and found the relationships of $V_{t}=0.21 Z^{0.34}$ and $V_{t}=0.91 Z^{0.16}$, where the former corresponds to the rain drops taken during the early development of the storm and the latter for the convective region. Ulbrich and Chilson (1994) theoretically investigated the effect of variations in rain drop size distribution and

Published by Copernicus Publications on behalf of the European Geosciences Union. 
fall speed law parameters on relation between mean Doppler fall speed and reflectivity factor. They suggested that the theoretical and/or empirical $V_{t}-Z$ relation may be used to estimate vertical wind velocity and particle size distribution, provided the shape of the size distribution and the phase of the precipitation particles are known a prior. Chu et al. (1999) analyzed precipitation data taken from 4 independent experiments conducted with the Chung-Li VHF radar and found that $\alpha$ and $\beta$ values in the power-law approximation $V_{t}=\alpha P^{\beta}$ above the melting layer are generally smaller than those below the layer, while in the height range of bright band the values of $\beta(\alpha)$ are significantly smaller (greater) than those above and below the bright band. A statistical power-law $V_{t}-Z$ relationship has been proposed by Protat et al. (2003) for the case of frontal cyclones to estimate terminal velocity of hydrometeors and the vertical wind velocity. Su et al. (2004) theoretically analyzed the power-law $V_{t}-P$ relation and showed that the mathematical relation between $\alpha$ and $\beta$ in the power-law approximation $V_{t}=\alpha P^{\beta}$ is in an exponential form, namely, $\alpha=A \exp (-\xi \beta)$, where $A$ is the coefficient in the relation $V=A D^{B}, V$ is fall speed of precipitation particle at diameter $D$, and $\xi$ is a factor related to radar parameters and precipitation intensity.

As mentioned before, the relation between $V_{t}$ and $P$ can be approximated to a power-law form. In order to avoid the air density effect on $V_{t}$ and range effect on $P$, the airdensity-corrected terminal velocity $\left(\rho_{0} / \rho\right)^{0.4} V_{t}$ and rangecorrected backscatter $P \times R^{2}$ should be used in establishing the $V_{t}-P$ relation, where $\rho_{0}$ and $\rho$ are, respectively, the air densities at ground and at certain height, $R$ is the range of the hydrometeors responsible for the backscatter $P$ (Beard, 1985). To the best of authors' knowledge, almost all existing $V_{t}-Z$ relations obtained through theoretical derivations or experimental analyses predict positive correlation between density-corrected $V_{t}$ and range-corrected $Z$ (or $P$ ) (Atlas et al., 1973; Chilson et al., 1993; Ulbrich and Chilson, 1994; Chu et al., 1999; Protat et al., 2003; Su et al., 2004; Su and $\mathrm{Chu}, 2007)$. One of the crucial assumptions made in developing these $V_{t}-Z$ relations is that no dynamic changes in the properties of the drop size distribution is considered, namely, the drop size distribution is assumed to be invariant within a few minutes. Moreover, one single theoretical distribution function is employed to model the drop size distribution to develop the $V_{t}-Z$ relations. Nevertheless, from the experimental results made with VHF wind profiler, a number of cases that show the negative correlations between $V_{t}$ and $P$ have been observed (Chu and Su, 1999; Chu et al., 1999; Su et al., 2004). That is, for the negative $V_{t}-Z$ correlation, the stronger the precipitation backscatter is, the smaller the magnitude of the terminal velocity will be. Experimental results also indicate that the height range where the negative $V_{t}-P$ relation prevails is usually around the melting layer that is characterized by substantially intense radar backscatter and dramatic increase of the terminal fall velocity of the melting hydrometeors with decreasing height. Lhermitte and
Atlas (1963) reported that, just below the bright-band peak, the precipitation-particles fall speed increases while the reflectivity decreases, implying a negative correlation between $V_{t}$ and $Z$. Obviously, the existing $V_{t}-Z$ relations that predict the positive correlation between $V_{t}$ and $Z$ cannot be applied to satisfactorily explaining the precipitation environment that generates the negative $V_{t}-Z$ correlation.

In this article, with the help of the Chung-Li $52 \mathrm{MHz}$ VHF radar located on the campus of National Central University in Taiwan, Republic of China, we present observational evidences that the negative $V_{t}-P$ relation not only occur in the melting layer (or bright band) region, but also may appear in the regions above and below the melting layer. One of the most important findings in this article is that the dynamic interaction of the precipitation particles between two different drop size distributions might significantly affect the $V_{t}-P$ correlation. In order to investigate the association of the interaction between precipitation particles with the $V_{t}-Z$ correlation, a scenario is proposed to account for the observed negative correlation between $V_{t}$ and $P$. Namely, the drop size distribution is assumed to be composed of two different Gamma drop size distributions with respective mean terminal velocities and radar reflectivities. The precipitation particles of these two Gamma distributions interact in the way that the number of the particles (or radar reflectivities) of each of the two distributions is changed. Analytical analysis shows that the negative correlation between $V_{t}$ and $Z$ occurs when the ratio of difference between relative changes in reflectivities of the two Gamma distributions to the total change in the radar reflectivity of the whole drop size distribution is positive. In Sect. 2, the experimental results obtained by the Chung-Li VHF radar are presented. In Sect. 3, theoretical relations between $V_{t}$ and $Z$ for the drop size distributions described by one and two Gamma distribution functions are discussed and developed, and the discussion is also given in this section. In Sect. 4, conclusion is drawn.

\section{Observations}

\subsection{Sensors and data descriptions}

The Chung-Li VHF radar operating at frequency of $52 \mathrm{MHz}$ (corresponding to $5.77 \mathrm{~m}$ wavelength) is located on the campus of National Central University in Taiwan (R.O.C.) $\left(24.9^{\circ} \mathrm{N}, 121^{\circ} \mathrm{E}\right.$, geomagnetic latitude $13.3^{\circ} \mathrm{N}$, Dip angle $35^{\circ} \mathrm{N}$ ). The whole antenna array of the radar consists of three independent sub-arrays. The first one is Mesosphere-Stratosphere-Troposphere (MST) sub-array that is used to measure atmospheric parameters and phenomena within height range from about 2 to $100 \mathrm{~km}$, including 3-dimensional wind field, turbulence, precipitation, gravity wave, and so on. The second one is ionospheric subarray that is employed to exclusively investigate spatial structures and dynamic behavior of electron density fluctuations 
in ionospheric E- and F-regions with interferometer capability. The third one is meteor sub-array for the determination of angle of arrival of the radar signals generated from meteor trail and lightning. Every sub-array consists of three independent and identical antenna modules. The MST sub-array with total antenna aperture $4800 \mathrm{~m}^{2}$ is used in this study to collect radar returns from precipitation and atmospheric refractivity fluctuations, which is arranged as an equal lateral triangle with length 45,45 , and $40 \mathrm{~m}$, respectively, and the apex is pointed toward north by west $22.3^{\circ}$. Each antenna module of the MST sub-array is a square array with 64 Yagi antenna elements $(8 \times 8)$ with a side length of $40 \mathrm{~m}$. The peak transmitted power of the Chung-Li radar is $120 \mathrm{kw}(3 \times 40 \mathrm{kw})$. Pulse repetition frequency (PRF) can be set from $1 \mathrm{~Hz}$ to $20 \mathrm{KHz}$. Maximum duty cycle of the transmitter is $7.5 \%$, and radar pulse width can be chosen arbitrarily in a range from 1 to $200 \mu$ s. Antenna beam width for each module of the MST sub-array is $7.4^{\circ}$ and $5^{\circ}$ for whole sub-array. The radar beams can be pointed not only toward zenith, but also toward northwest, northeast, southeast and southwest at fixed zenith angle $17^{\circ}$ such that three-dimensional wind field can be measured.

An optical rain gauge implemented at the Chung-Li VHF radar site is employed to record surface rainfall rate. The dynamic range of this rain gauge is from 0.5 to $1600 \mathrm{~mm} \mathrm{~h}^{-1}$. The analogous output of the recorded data is sampled at a rate of $0.1 \mathrm{~Hz}$ (corresponding to $10-\mathrm{s}$ time resolution) and stored in an external hard disk for further processing. The optical rain gauge should be operated in a temperature range of $0^{\circ}-$ $50^{\circ}$ to maintain recoded data quality.

The radar data employed in this article for the investigation of the $V_{t}-P$ relationship was taken on 18 July 2000, from about 15:44 to 19:52 LT. Radar parameters employed for precipitation experiment were given in Table 1. The Doppler spectra of the radar returns are calculated by using 128-point FFT algorithm. Three raw spectra are averaged incoherently to produce an average spectrum for further analysis, resulting in a Doppler velocity spectral resolution of $0.313 \mathrm{~m} \mathrm{~s}^{-1}$. The properties of the radar returns are also described in Table 1. The ground-clutter signals are removed from the radar returns by filtering out the DC component of the radar echoes. We note that the radar returns from precipitation and refractivity fluctuations can be separated only in the spectral domain, rather than in time domain. It is obvious that, for vertically pointing radar beam, there is a tendency for precipitation echoes to locate in spectral domain with positive Doppler frequency shift due to the falling of hydrometeors. Note that the positive (negative) Doppler frequency shift represents the target moving toward (away) the radar. However, in an environment with strong updraft with speed greater than the hydrometeor falling velocity, the spectral components of the precipitation may be located in the negative Doppler frequency domain. The locations of the spectral component for refractivity fluctuations are quite variable, depending on the direction of vertical air velocity. Therefore, the spectral
Table 1. Radar parameters and radar signal properties for precipitation experiment.

\begin{tabular}{lc}
\hline Parameter & value \\
\hline Pulse Width & $2 \mu \mathrm{s}$ \\
Range Resolution & $300 \mathrm{~m}$ \\
Inter-Pulse Period & $300 \mu \mathrm{s}$ \\
Number of Coherent Integration & 240 \\
Time resolution of radar data & $0.072 \mathrm{~s}$ \\
Steering of antenna beam & $0^{\circ}($ zenith $)$ \\
Height coverage & $1.8-13.5 \mathrm{~km}$ \\
Number of range gate & 40 \\
Point of FFT algorithm & 128 \\
Doppler Spectral Resolution & $0.313 \mathrm{~m} \mathrm{~s}^{-1}$ \\
Number of Incoherent Integration & 3 \\
\hline
\end{tabular}

components for precipitation and turbulent refractivity can be separated unambiguously in the Doppler spectral domain as long as the radar parameters are set appropriately and the atmospheric turbulences are not so active that the spectral components are capable of being clearly identified. The echo power, mean Doppler frequency shift and spectral width for these two spectral components are estimated separately with the least square method, in which the Gaussian curve is employed to best fit the corresponding Doppler spectral components. In light of the fact that observed mean Doppler velocity of precipitation particles is the sum of terminal fall velocity of the hydrometeors and vertical air velocity which can be estimated from the spectral component of turbulent refractivity, the terminal velocity can thus be obtained by removing the vertical air velocity from the observed Doppler velocity of the precipitation. In addition, the observed spectral width of the precipitation echoes is the result of the convolution of the precipitation particle spectrum with clearair Doppler spectrum, leading to the observed precipitation echoes are subjected to beam broadening effect (Chu et al., 1991). Therefore, the deconvolution of the observed precipitation spectrum should be required to conduct to obtain true spectral width caused by precipitation particles.

\subsection{Observational results}

Figure 1 shows two examples of height variations of observed Doppler spectra, in which the dashed lines superimposed on the spectral components of the precipitation with negative Doppler velocity represent the trend of the height variations of the precipitation Doppler velocities. As indicated, relatively intense precipitation backscatter are seen in the height range about $4.5-5 \mathrm{~km}$, which is coincident with the level of $0^{\circ} \mathrm{C}$ isotherm at height about $4.8 \mathrm{~km}$ measured by rawinsonde at Pan-Chiao about $25 \mathrm{~km}$ northeast from the Chung-Li VHF radar site. This feature suggests that the enhancement of the radar backscatter in height range $4.5-5 \mathrm{~km}$ is the bright band structure that is associated with the melting 
18:39:11 - 18:39:38 LT

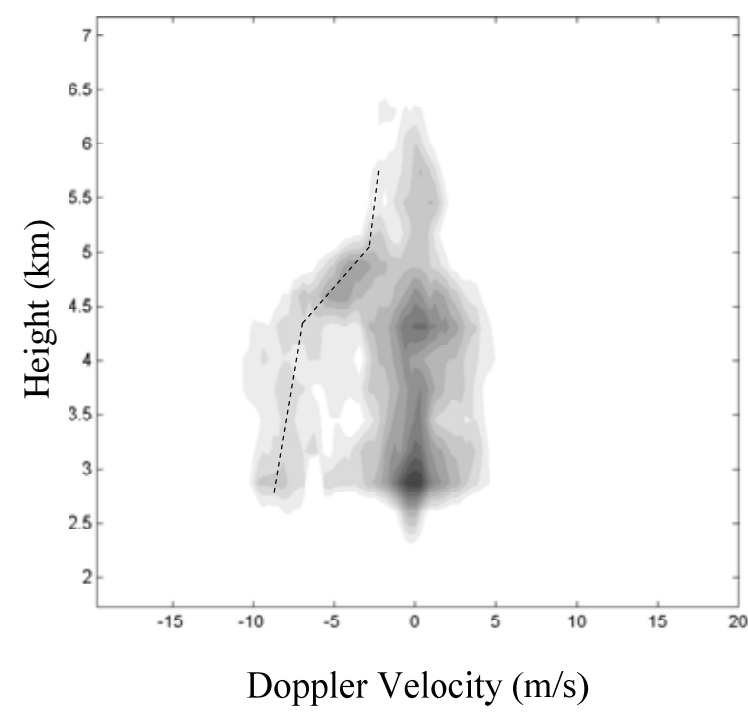

$\mathrm{dB}$

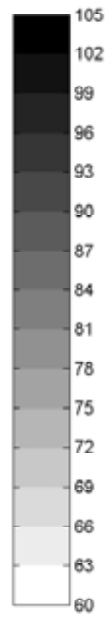

19:13:18 - 19:13:45 LT

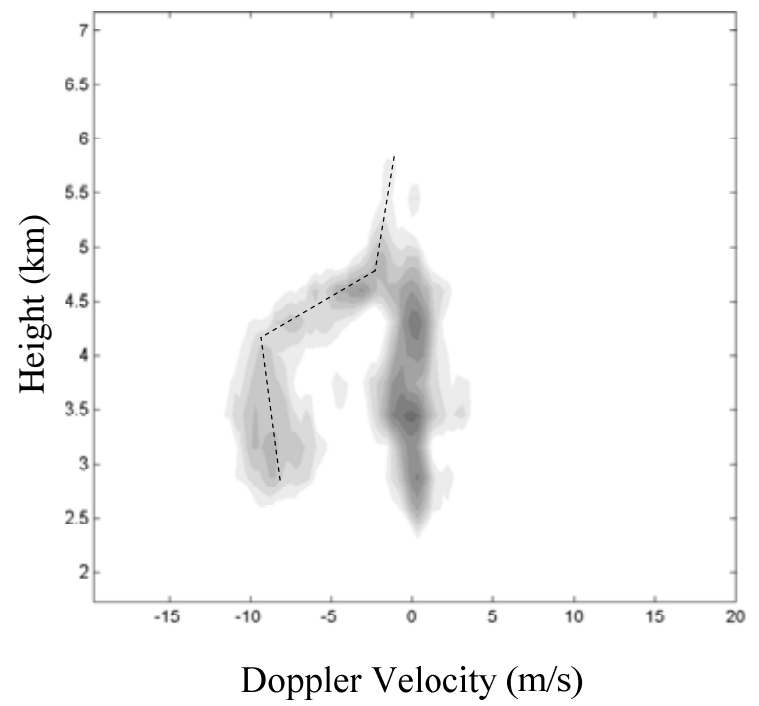

$\mathrm{dB}$

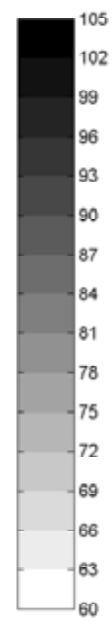

Fig. 1. Examples of height variation of radar-observed Doppler spectra, in which dashed lines represent the height variations in precipitation Doppler velocities with a height resolution of $300 \mathrm{~m}$. The grey scale represents Doppler spectral density expressed in decibels of arbitrary unit per velocity bin. Right and left panels show different trends of height variations in precipitation Doppler velocities.

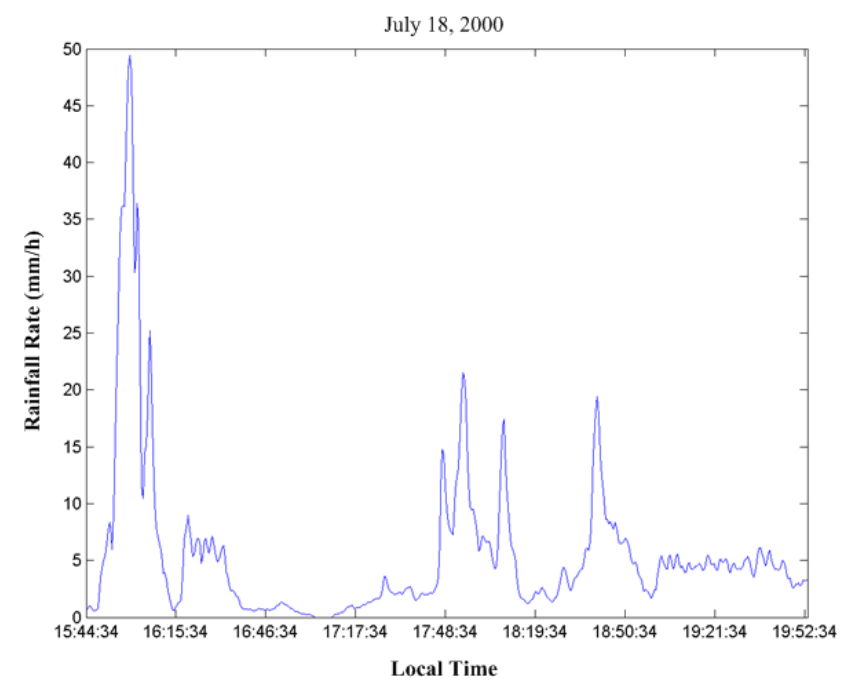

Fig. 2. Time series of rain fall rate recorded on 18 July 2000, by optical rain gauge collocated with the Chung-Li VHF radar, which continuously functioned to collect surface rainfall rate.

hydrometeors which are falling in the melting layer. Figure 1 also shows that the mean terminal velocities of the hydrometeors above and below the melting layer are, respectively, about $2-3 \mathrm{~m} \mathrm{~s}^{-1}$ and $8-10 \mathrm{~m} \mathrm{~s}^{-1}$. It is noteworthy from Fig. 1 that the terminal velocity of the hydrometeors above the melting layer shows a gradual increase with decreasing height. However, the height variation of the terminal velocity for the rain drops below the melting layer is variable, it may increase (as shown in left panel) or decrease (as shown in right panel) with decreasing height.

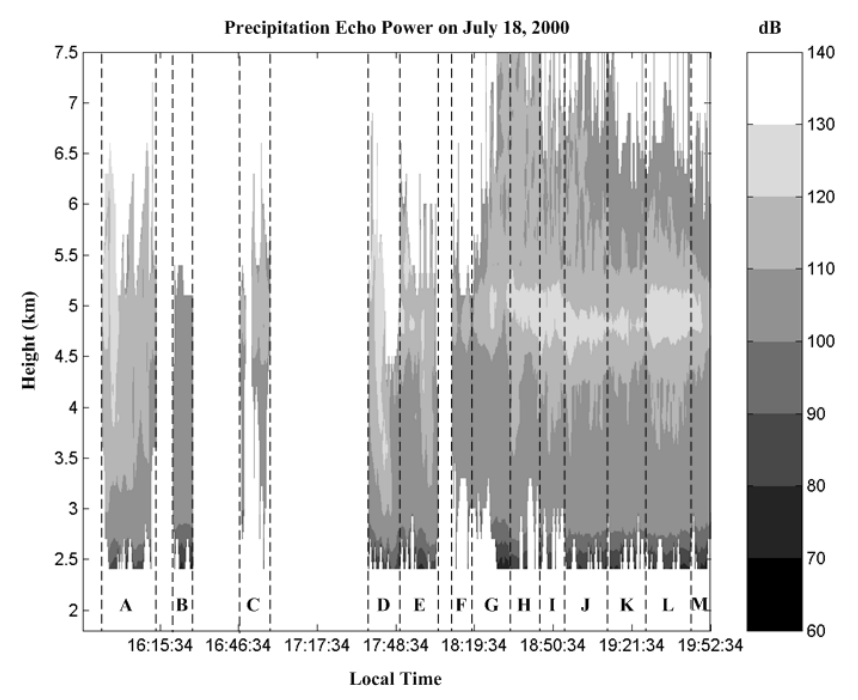

Fig. 3. Height-time contour plot of VHF echo power from precipitation particles.

Figure 2 is the time sequence of rain fall rate measured by ground-based optical rain gauge co-located with the Chung$\mathrm{Li}$ radar. There are a number of intense and intermittent precipitation events occurred in periods 15:46-16:14 LT, 17:47-18:18 LT, and 18:38-18:54 LT. There are also relatively steady precipitation event occurred in periods 16:1616:34 LT and 19:00-19:46 LT. Note that it is very difficult to distinguish convective or stratiform precipitations only from the record of the surface rain fall rate without other information. In fact, with the help of radar-measured profiles 


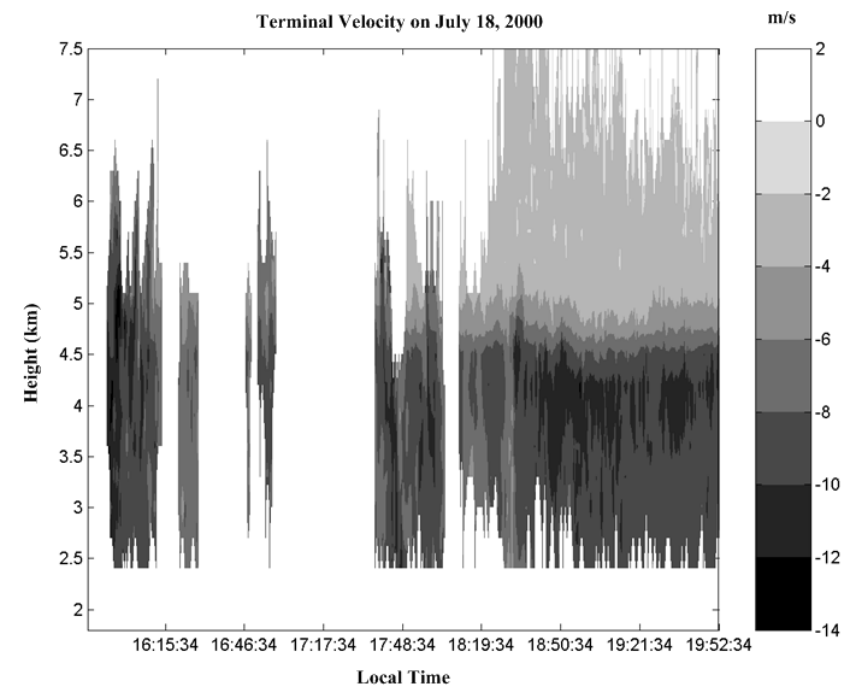

Fig. 4. Height-time contour plot of Doppler velocities of precipitation particles.

of terminal velocity and precipitation echo power presented later, the intense precipitation event in the period 16:1616:34 LT is believed to be categorized into convective precipitation, and that in the period 18:38-18:54 LT is thought to attribute to the stratiform precipitation.

Figures 3, 4 and 5 present height-time contour plots for precipitation echo power, terminal velocity of precipitation particles, and vertical air velocity, respectively. As shown in Figs. 3 and 4, in the period from 18:10 to 19:52 LT a continuous and stable bright band structure accompanied by abrupt change in terminal velocity in height range from 4.5 to $5 \mathrm{~km}$ is clearly seen. Moreover, as indicated in Fig. 5, during this period the weak and negative (in the downward direction) vertical air velocity with the maximum value less than $2.5 \mathrm{~m} \mathrm{~s}^{-1}$ prevails below the melting layer and its height-time distribution is much less organized. However, the vertical air velocity above the melting layer seems to be primarily upward, especially above the height about $5.5 \mathrm{~km}$. Presumably, this upward air motion is likely to be the result of latent heat release of stratiform cloud droplets through condensation/deposition process (Houze, 1993). The existence of the bright band structure combined with the pattern of the height variation of the terminal velocity above and below the melting layer suggest that the rain fall occurred in this period is categorized into the stratiform (or cold) precipitation which is originated from the small hydrometeors above the level of $0^{\circ} \mathrm{C}$ isotherm and then falls through the melting layer to form large rain drop. However, the precipitations occurred before 17:49 LT are very different from those after 17:49 LT. Figures 3 and 4 demonstrate that the patterns of the height variations of the terminal velocity and backscatter for the precipitation events before 17:49 LT are not only intermittent and short-lived, but also very irregular and unsystematic

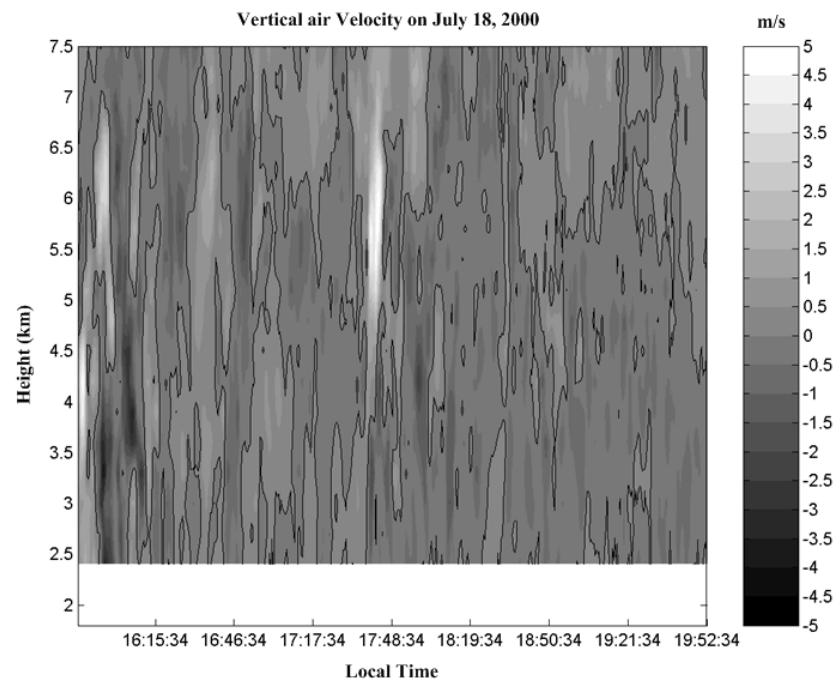

Fig. 5. Height-time contour plot of vertical air velocity.

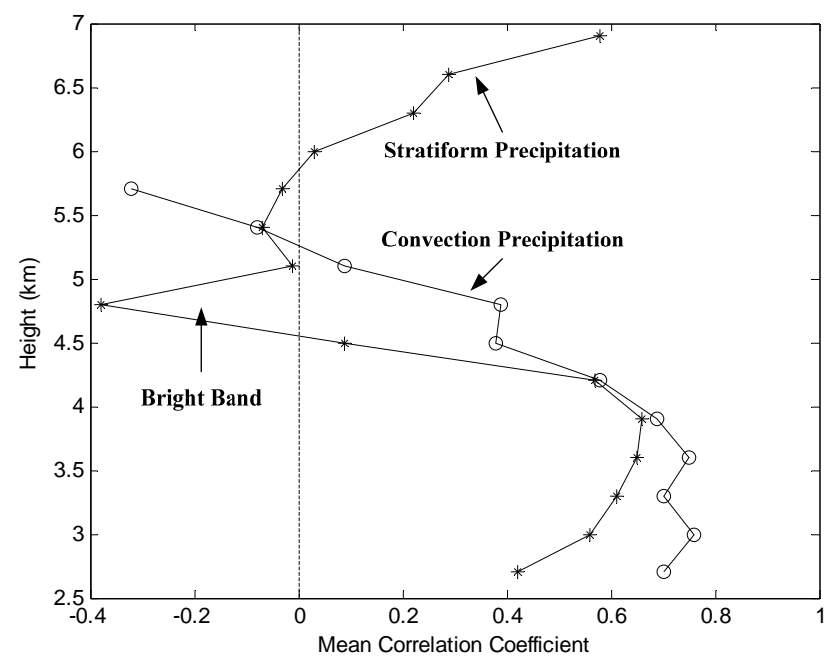

Fig. 6. Profiles of mean correlation coefficients between airdensity-corrected terminal velocity and range-corrected echo power for convective and stratiform precipitations as shown in Figs. 3 and 4 .

compared to the case of the stratiform precipitation, in which no bright band structure is found. We also note from Fig. 5 that very strong updrafts with maximum magnitude greater than $5 \mathrm{~m} \mathrm{~s}^{-1}$ occur at the time when the intense rain falls are observed. This one-to-one correspondence between strong vertical air velocity and intense precipitation event is a signature of convective precipitation.

Figure 6 shows the height variations of the mean correlation coefficients between air-density-corrected terminal velocity and range-corrected backscatter for the convective (before 17:49LT) and stratiform (after 17:49LT) precipitations, which are taken logarithms for the base 10 prior to calculating the correlation coefficient that is given by 

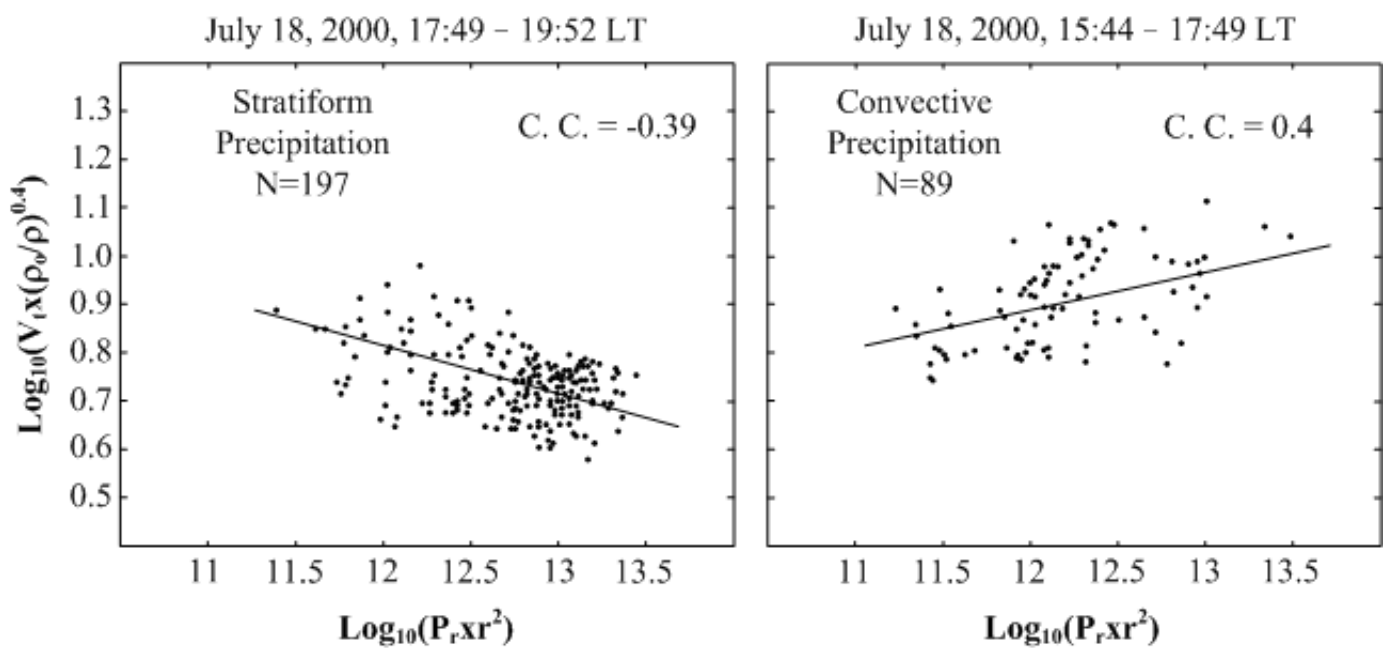

Fig. 7. Examples of scatter diagrams of density-corrected terminal velocity versus range-corrected precipitation echo power for stratiform (left panel) and convective (right panel) precipitations at height $4.8 \mathrm{~km}$, in which correlation coefficient (C.C.) between the terminal velocity and echo power for convective and stratiform precipitations are displayed.

Table 2. List of period, number of radar measured precipitation profiles and rain type for different precipitation events.

\begin{tabular}{cccc}
\hline Event & Period & Number of profile & Rain type \\
\hline A & $15: 52-16: 13$ LT & 47 & Convective \\
B & $16: 21-16: 30$ LT & 21 & Convective \\
C & $16: 46-17: 00$ LT & 32 & Convective \\
D & $17: 37-17: 49$ LT & 27 & Convective \\
E & $17: 49-18: 05$ LT & 35 & Stratiform \\
F & $18: 10-18: 19$ LT & 21 & Stratiform \\
G & $18: 19-18: 33$ LT & 31 & Stratiform \\
H & $18: 33-18: 45$ LT & 27 & Stratiform \\
I & $18: 45-18: 55$ LT & 22 & Stratiform \\
J & $18: 55-19: 11$ LT & 36 & Stratiform \\
K & $19: 11-19: 27$ LT & 35 & Stratiform \\
L & $19: 27-19: 45$ LT & 40 & Stratiform \\
M & $19: 45-19: 52$ LT & 17 & Stratiform \\
\hline
\end{tabular}

$C_{X Y}=\frac{\sum_{i}\left(x_{i}-\mu_{X}\right)\left(y_{i}-\mu_{Y}\right)}{\sigma_{X} \sigma_{Y}}$

where $x_{i}$ and $y_{i}$ represent the values of the random variables of $X$ and $Y$, respectively, $\mu_{X}$ and $\mu_{Y}$ are mean values and $\sigma_{X}^{2}$ and $\sigma_{Y}^{2}$ are variances of $X$ and $Y$, respectively. As shown, for the stratiform precipitation the peak of the negative correlation is primarily located in the height range around the bright band and the positive correlations are seen in the regions below and above the melting region. As for the convective precipitation the negative (positive) correlation occurs above (below) the melting layer. The scatter diagrams of airdensity-corrected terminal velocity versus range-corrected precipitation echo power at height 4.8 for convective and stratiform precipitations are, respectively, shown in Fig. 7, in which the straight lines superimposed on the respective scatter data points are the lines best fitted to the data using the least-squares method. It is clear from Fig. 7 that the observed relation between the logarithms of the density-corrected $V_{t}$ and the range-corrected $P$ primarily follow a straight line, despite the correlation coefficient. In fact, the linear dependence between logarithms of $V_{t}$ and $P$ is the direct result of the power-law relationship between $V_{t}$ and $P$ (or $Z$ ) that has been validated theoretically and experimentally (Ulbrich and Chilson, 1994; Chu et al., 1999).

The height variations of the $V_{t}-P$ correlation presented in Fig. 6 is the result of long-term (longer than $120 \mathrm{~min}$ ) statistics. Questions arise as to whether or not the $V_{t}-P$ correlation for a precipitation event occurred in relatively short period (typically between $10-30 \mathrm{~min}$ ) may also remain significantly negative, and what is the precipitation environment or physical process which favor the occurrence of the negative $V_{t}-P$ correlation. In order to answer these questions, we divide the radar-measured precipitation data into 13 segments in accordance with the temporal variations in the precipitation backscatter and terminal velocity aloft combined with surface rain fall intensity as shown in Fig. 2. The periods of the 13 segmented precipitation events are shown in Table 2. Figure 8 shows the time sequence of the mean terminal velocity profiles of the 13 precipitation events, in which open square and solid circles on the terminal velocity profiles represent positive and negative correlations between $V_{t}$ and $P$, respectively. Note that the profile marked with $M$ (corresponding to the precipitation event $\mathrm{M}$ defined in Table 2) on the right side of Fig. 8 is chosen as the reference profile that should be read based on the scale shown in abscissa. The other profiles are plotted in accordance with the sequence of the precipitation events with a separation of $5 \mathrm{~m} \mathrm{~s}^{-1}$ between 


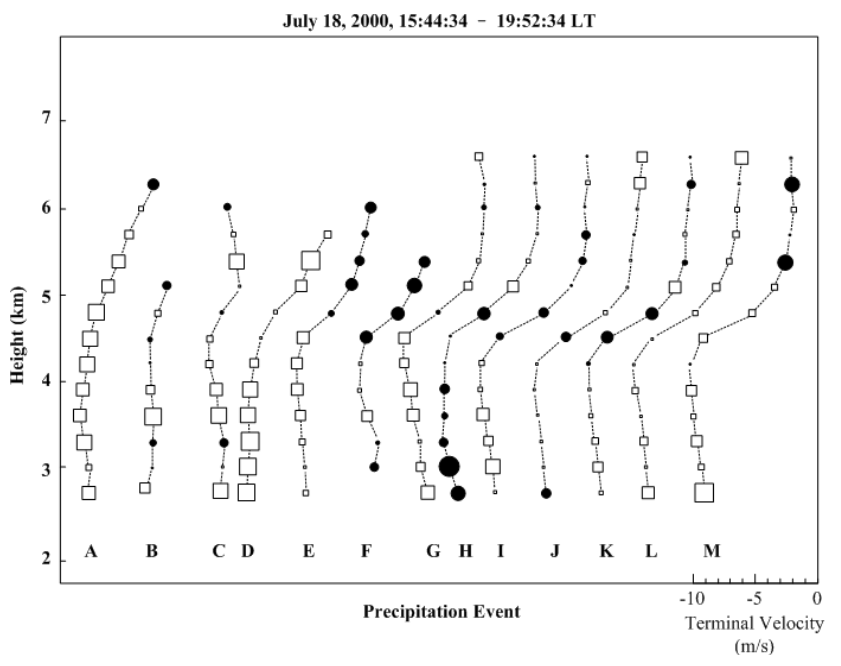

Fig. 8. Height-time variations of mean terminal velocities of precipitation particles, in which open squares and solid black dots in the mean profiles represent, respectively, positive and negative correlations between density-corrected terminal velocity and rangecorrected echo power. The sizes of the symbols reflect the magnitudes of the correlation coefficients. Period of each precipitation event can be referred to Table 2 . Note that negative terminal velocity corresponds to downward motion of the precipitation particles.

two adjacent profiles. As expected from the long-term statistic result, for the precipitations below the melting layer the $V_{t}-P$ correlations presented in Fig. 8 in all likelihood are positive and only very limited cases possess negative $V_{t}-P$ correlations. Examining the stratiform precipitation shown in Fig. 8 in more detail indicates that the negative $V_{t}-P$ correlation seems to prefer to occur in the lower part of the bright band region that is characterized by abrupt increase in the terminal velocity and dramatic decrease in the radar reflectivity with decreasing height. Moreover, except for the bright band region, the negative $V_{t}-P$ correlations can also be found in the height ranges above and below the melting layer where no significant changes in the terminal velocity and radar reflectivity with height are seen. In next section, a scenario is proposed to theoretically investigate in what precipitation environment that favors the occurrence of the negative $V_{t}-Z$ correlation.

Figure 9 presents the height variation of the $\beta$ values in the power-law relation $\left(\rho_{o} / \rho\right)^{0.4} V_{t}=\alpha\left(P \times R^{2}\right)^{\beta}$ for convective and stratiform precipitations, respectively. As indicated, the $\beta$ values for the convective and stratiform precipitations below the melting layer (about $4.5 \mathrm{~km}$ ) are very similar, with a magnitude varied between $0.08-0.13$. This magnitude is slightly smaller than those obtained by Chilson et al. (1993) and Protat et al. (2003) (about 0.16). However, within and above the melting layer, the trend of the height variation of the $\beta$ value for the stratiform precipitation is opposite to that for the convective precipitation. Presumably, the cause of the

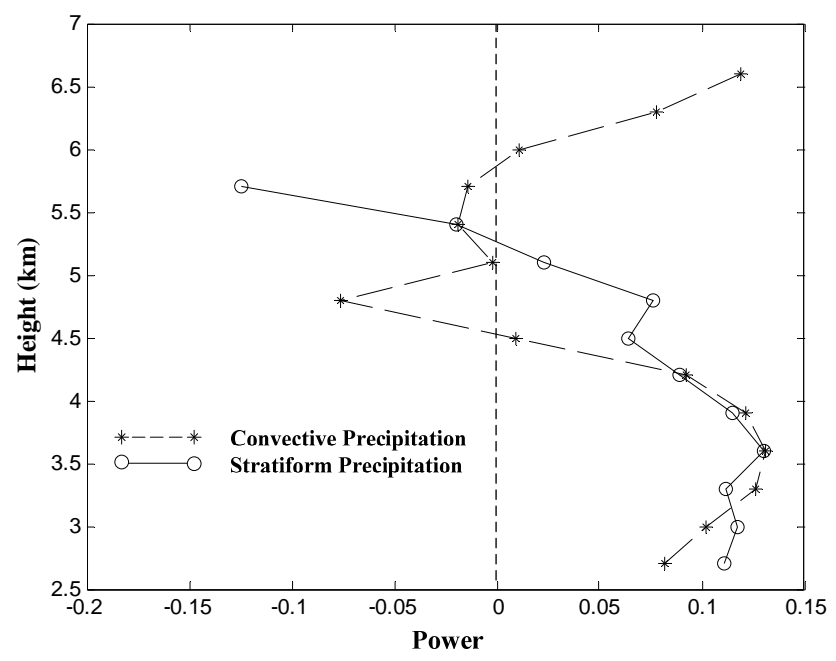

Fig. 9. Height variation of the magnitude of the $\beta$ values in the power-law relation $\left(\rho_{o} / \rho\right)^{0.4} V_{t}=\alpha\left(P \times R^{2}\right)^{\beta}$ for convective and stratiform precipitations.

difference in the two trends is very likely attributed to the different precipitation processes.

\section{Theoretical explanation and discussion}

\subsection{Background}

Theoretical studies of the terminal velocity-backscatter relation has been made by a number of investigators for years (Atlas et al., 1973; Chilson et al., 1993; Ulbrich and Chilson, 1994; Chu et al.,1999; Protat et al., 2003; Su et al., 2004; Su and Chu, 2007). To the best of authors' knowledge, almost all of the existing $V_{t}-Z$ (or $V_{t}-P$ ) relations are developed by assuming a drop size distribution modeled by a theoretical distribution function combined with a theoretical fall speeddiameter relation. The Gamma drop size distribution (DSD) has been widely employed in the community of radar meteorology to model observed raindrop size distributions (Ulbrich, 1983; Kozu and Nakamura, 1991; Su and Chu, 2007). The expression of the Gamma DSD is given by

$N(D)=N_{0} D^{\mu} e^{-\delta D}$,

where $N(D)$ is the drop size distribution $\left(\mathrm{m}^{-3} \mathrm{~mm}^{-1}\right), D$ is the drop diameter $(\mathrm{mm}), N_{0}$ is the number density parameter $\left(\mathrm{m}^{-3} \mathrm{~mm}^{-1-\mu}\right), \delta$ is the slope parameter $\left(\mathrm{mm}^{-1}\right)$ and $\mu$ is a dimensionless shape parameter. The fall speed-diameter relations that are commonly employed for the development of the $V_{t}-Z$ relation are generally modeled by the power-law and empirical approximations that are, respectively, given by (Foote and du Toit, 1969)

$V(D)=A D^{B}$ 


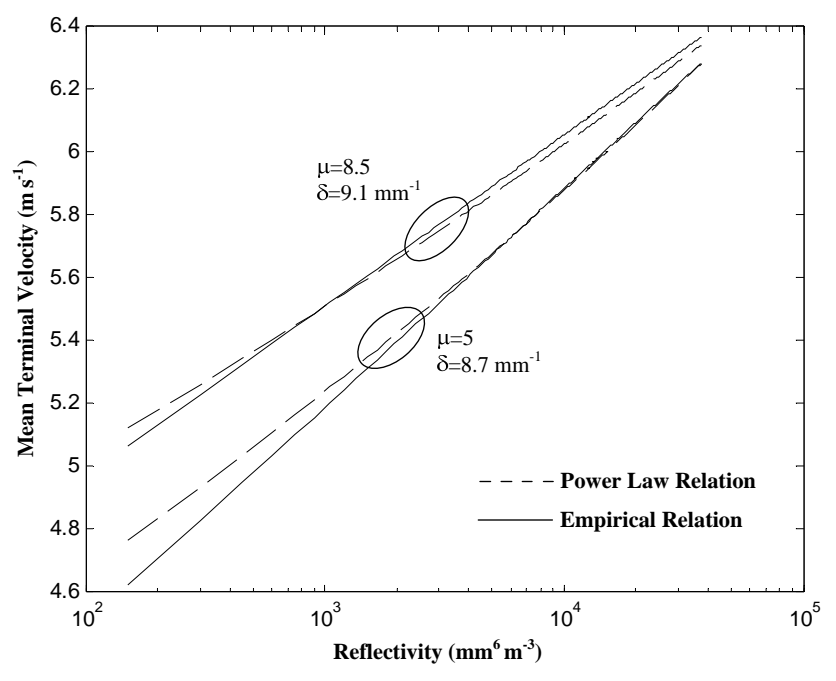

Fig. 10. Examples of $V_{t}-Z$ relation for one single Gamma drop size distribution, in which the power-law and exponential relations are, respectively, corresponding to expressions (6) and (7). The parameters used for the calculations, see text.

$V(D)=9.65-10.3 e^{-0.6 D}$

Especially note that the unit of the drop diameter $D$ in Eq. (2) is cm, while that in Eq. (3) is mm. The definitions of the radar reflectivity $\mathrm{Z}$ and the reflectivity-weighted terminal velocity $V_{t}$ are, respectively, given by

$Z=\int N(D) D^{6} d D$

$V_{t}=\frac{\int V(D) N(D) D^{6} d D}{\int N(D) D^{6} d D}$

Substituting Eqs. (1), (2) and (3) into Eqs. (6) and (7) and performing mathematical manipulations, we obtain two theoretical $V_{t}-Z$ relations given by (Atlas et al., 1973; Ulbrich and Chilson, 1994; $\mathrm{Su}$ and Chu, 2007)

$$
\begin{aligned}
V_{t} & =\frac{A \Gamma(B+\mu+7)}{N_{o}^{B /(\mu+7)}[\Gamma(\mu+7)]^{(B+\mu+7) /(\mu+7)}} Z^{B /(\mu+7)} \\
V_{t} & =9.65-10.3 \times\left[1+0.6\left(\frac{Z}{N_{0} \Gamma(\mu+7)}\right)^{1 /(\mu+7)}\right]^{-(\mu+7)} \\
& =9.65-10.3 \times\left(1+\frac{0.6}{\delta}\right)^{-(\mu+7)}
\end{aligned}
$$

where expressions (6) and (7) correspond to the fall speeddiameter relations of power-law form (expression 2) and empirical approximation (expression 3), respectively. Note that, irrespective of their complicate forms, the theoretical expressions of Eqs. (6) and (7) predict a positive dependence of $V_{t}$ on $Z$, or equivalently, a positive correlation between $V_{t}$ and $Z$ (Atlas et al., 1973; Ulbrich and Chilson, 1994; Chu et al.,1999; Su et al., 2004). In order to realize the $V_{t}-Z$ relations described by Eqs. (6) and (7), the parameters No is estimated in accordance with the following expression

$N_{0}=\frac{M \delta^{\mu+1}}{\Gamma(\mu+1)}$

where $M$ is the total number of precipitation particles in unit volume and its definition is given by

$M=\int N(D) d D$

Therefore, Eq. (8) is easily obtained by substituting Eq. (1) into Eq. (9) and performing the mathematical integration. Figure 10 presents examples of $V_{t}-Z$ relations, in which dashed and solid curves are corresponding to the expressions of Eqs. (6) and (7), respectively. The parameters employed for the calculations are that: $A=16.9 \mathrm{~m} \mathrm{~s}^{-1}, B=0.6$, $M=750 \mathrm{~m}^{-3}, \mu=8.5$ and $5, \delta=9.1$ and $8.7 \mathrm{~mm}^{-1}$ (Sekhon and Srivastava, 1971; Chu and Su, 2008). As shown in Fig. 10, a positive dependence of $V_{t}$ on $Z$ is expected. Obviously, the physics behind these two expressions fail to account for the precipitation environment favorable to the formation of the negative $V_{t}-P$ correlation observed by the VHF radar, especially in the bright band region that contains melting and melted hydrometeors which are believed to interact dynamically to result in abrupt changes in terminal velocity and radar reflectivity (Battan, 1973). This feature strongly suggests that the interaction of the precipitation particles with different sizes and terminal velocities is very likely to play a crucial role in governing the correlation between $V_{t}$ and $Z$. We therefore propose a scenario of a bimodal drop size distribution in next subsection to theoretically explore in what conditions the correlation between $V_{t}$ and $Z$ will be negative or positive.

\subsection{Analytical analysis of $d V_{t} / d Z$}

As underscored in Sect. 3.1, the drop size distribution modeled by one single Gamma distribution function cannot satisfactorily explain the negative $V_{t}-Z$ relation. In order to solve this problem, we propose that the drop size distribution is the linear superposition of two Gamma drop size distributions, namely,

$N(D)=N_{1}(D)+N_{2}(D)$

where

$N_{1}(D)=N_{01} D^{\mu_{1}} e^{-\delta_{1} D}$

$N_{2}(D)=N_{02} D^{\mu 2} e^{-\delta_{2} D}$

Under this condition, the corresponding mean terminal velocity of $N(D)$ is estimated by

$V_{t}=\frac{\int V(D)\left(N_{1}(D)+N_{2}(D)\right) D^{6} d D}{\int\left(N_{1}(D)+N_{2}(D)\right) D^{6} d D}$ 


$$
=\frac{V_{t 1} Z_{1}+V_{t 2} Z_{2}}{Z_{1}+Z_{2}}
$$

where $V_{t 1}$ and $V_{t 2}$ are, respectively, the mean terminal velocities of the precipitation particles with the drop size distributions of $N_{1}(D)$ and $N_{2}(D), Z_{1}$ and $Z_{2}$ are, respectively, their reflectivities, and the total reflectivity $Z$ is equal to the sum of $Z_{1}$ and $Z_{2}$. Note that the correlation between $V_{t}$ and $Z$ bears a close relation to the ratio of the change in $V_{t}$ to that in $Z$, namely, $d V_{t} / d Z$. To proceed, the differential form of Eq. (13) is given by

$d V_{t}=\frac{\left(V_{t 2}-V_{t 1}\right)\left(Z_{1} d Z_{2}-Z_{2} d Z_{1}\right)}{\left(Z_{1}+Z_{2}\right)^{2}}+\frac{Z_{1} d V_{t 1}+Z_{2} d V_{t 2}}{\left(Z_{1}+Z_{2}\right)}$

Dividing Eq. (14) by $d Z$ reduces to

$\frac{d V_{t}}{d Z}=\frac{Z_{1} Z_{2}\left(V_{t 2}-V_{t 1}\right)}{Z^{2}} \Delta+\frac{1}{Z} \Pi$

where

$\Delta=\frac{\left(d \ln Z_{2}-d \ln Z_{1}\right)}{\left(d Z_{1}+d Z_{2}\right)}$

$\Pi=\frac{Z_{1} d V_{t 1}+Z_{2} d V_{t 2}}{\left(d Z_{1}+d Z_{2}\right)}$

From Eqs. (14), (15) and (16), we note that a number of factors affect the sign and magnitude of $d V_{t} / d Z$, including difference between $V_{t 1}$ and $V_{t 2}$, difference between relative changes in $Z_{1}$ and $Z_{2}$ (i.e., $d \ln Z_{2}-d \ln Z_{1}$ ), change in total reflectivity $d Z\left(=d Z_{1}+d Z_{2}\right)$, and the sum of $Z_{1} d V_{t 1}$ and $Z_{2} d V_{t 2}$. We will show later that $\Delta$ defined in Eq. (15) plays the most significant role in dominating the sign and magnitude of $d V_{t} / d Z$, or equivalently the correlation between $V_{t}$ and $Z$.

In order to realize in what conditions that the sign of $d V_{t} / d Z$ will become negative, we make an extensive calculation survey by giving various pairs of scale and shape parameters and drop densities, i.e., $\delta_{1}, \delta_{2}, \mu_{1}, \mu_{2}, M_{1}$ and $M_{2}$, in $N_{1}(D)$ and $N_{2}(D)$ to obtain respective terminal velocities and reflectivities. The empirical fall speed-diameter relation, i.e., expression (3), is adopted to calculate the terminal velocity in accordance with Eq. (11). Note that the experimental results shown in Sect. 2 indicate that the observed negative correlation between $V_{t}$ and $Z$ predominately prevails in the bright band region characterized by abrupt increase in the terminal velocity with decreasing height. This feature is an implication of the dynamic interaction between melting and melted hydrometeors in the melting region. In order to simulate the interaction of the precipitation particles between two different kinds of the drop size distributions, we first give appropriate values of $\delta_{1}, \delta_{2}, \mu_{1}$ and $\mu_{2}$ in $N_{1}(D)$ and $N_{2}(D)$ to make the mean terminal velocity of $N_{1}(D)$ slightly greater than those of $N_{2}(D)$. In addition, the initial values of $M_{1}$ and $M_{2}$ are specified in such way that the corresponding $Z_{1}$ is weaker than $Z_{2}$, which are 500 and $2000 \mathrm{~m}^{-3}$, respectively. We then further assume that $M_{2}$ is reduced with

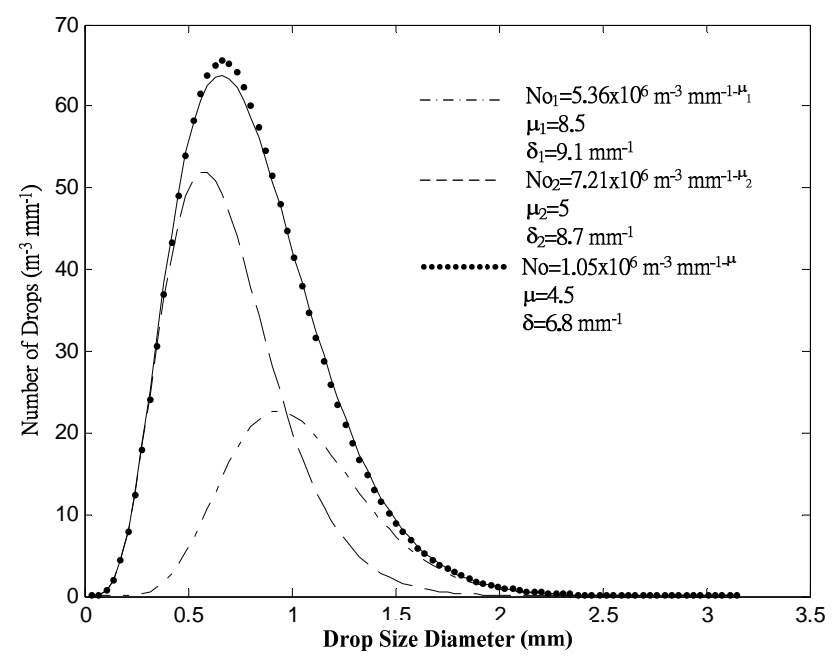

Fig. 11. Simulated drop size distribution (solid curve) composed of two continuous Gamma distribution functions $N_{1}(D)$ and $N_{2}(D)$ with different scale and slope parameters. Dotted curve is the best fitted Gamma distribution function to the simulation result. The total number of precipitation particles in unit volume for the distributions of $N_{1}(D)$ (dot-dashed curve) and $N_{2}(D)$ (dashed curve) are, respectively, 500 and $2000 \mathrm{~m}^{-3}$.

time and $M_{1}$ is accordingly increased to signify the interaction of the precipitation particles between $N_{1}(D)$ and $N_{2}(D)$. The changes in $M_{2}$ and $M_{1}$ will lead to the corresponding changes in $Z_{1}$ and $Z_{2}$ in accordance with Eqs. (4) and (8), but they will not cause the changes in $V_{t 1}$ and $V_{t 2}$ if the scale and shape parameters of the Gamma drop size distributions are kept constant as shown in Eq. (7). As a result, the magnitude of $d V_{t} / d Z$ can be eventually obtained in accordance with Eqs. (14-16). In practical computation, the continuous interaction between the precipitation particles is modeled by a discrete sequence of $M_{1}$ and $M_{2}$. The small amounts of the changes in $M_{1}$ and $M_{2}$ in the respective discrete sequences are carefully specified so that the corresponding values of $d V_{t 1}, d V_{t 2}, d Z_{1}, d Z_{2}$ and $d V_{t} / d Z$ can be numerically estimated.

Figure 11 is an example of simulated drop size distribution (continuous curve) that is the composition of two continuous Gamma distribution functions $N_{1}(D)$ (dot-dashed curve) and $N_{2}(D)$ (dashed curve) which are characterized by the parameters of $N_{01}, N_{02}, \delta_{1}, \delta_{2}, \mu_{1}$ and $\mu_{2}$ with values given in the figure. The values of $M_{1}$ and $M_{2}$ employed for the computing $N_{01}$ and $N_{02}$ are, respectively, given by 500 and $2000 \mathrm{~m}^{-3}$. The mean terminal velocities of $N_{1}(D)$ and $N_{2}(D)$ are, respectively, $5.83 \mathrm{~m} \mathrm{~s}^{-1}$ and $5.02 \mathrm{~m} \mathrm{~s}^{-1}$. As shown in Fig. 11, for the parameters given above, the simulated drop size distribution bears a strong resemblance to a Gamma distribution function, in which the dotted curve is the best fitted Gamma function to the simulated drop size distribution according to the least-squares method. It is clear to see that the simulated 

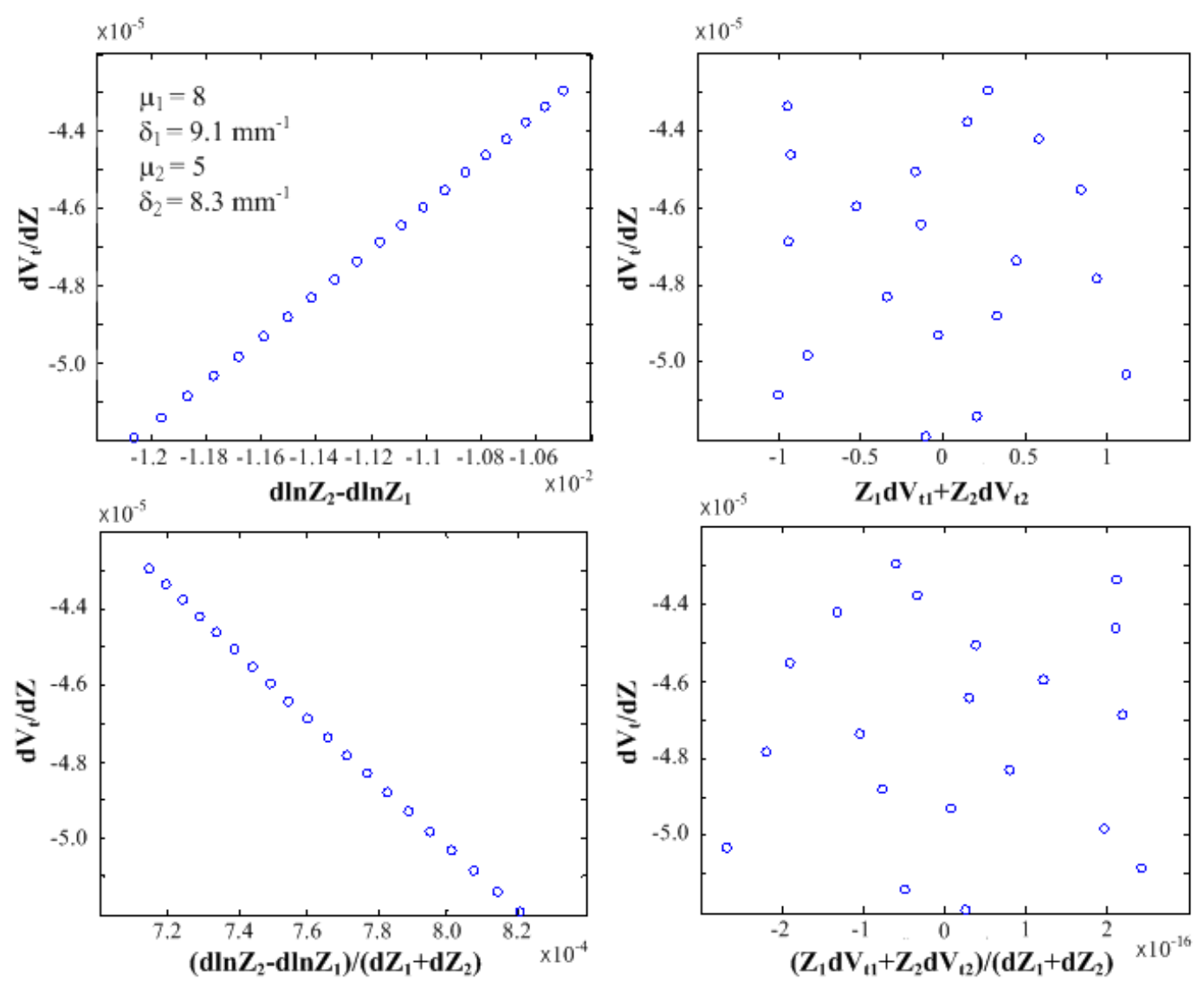

Fig. 12. Dependence of $d V_{t} / d Z$ on difference factors that correspond to expressions (14-16) in text.

and best fitted drop size distributions are almost identical to each other, except for the portion around the peak of the distributions. This example indicates that it is very difficult to distinguish the components of the drop size distributions that constitute an observed drop size distribution from its appearance. Therefore, the conventional way of modeling an observed drop size distribution by using one Gamma distribution function may not be adequate.

The expressions (14-16) show that the sign and magnitude of $d V_{t} / d Z$ is a function of the factors $V_{t 2}-V_{t 1}, d \ln Z_{2}-$ $d \ln Z_{1}, d Z_{1}+d Z_{2}$, and $Z_{1} d V_{t 1}+Z_{2} d V_{t 2}$. The value of $V_{t 2}-$ $V_{t 1}$ is a constant as long as the scale and shape parameters of the Gamma distribution functions are constants. For the drop size distribution as shown in Fig. 11, the value of $V_{t 2}-V_{t 1}$ is equal to $-0.81 \mathrm{~m} \mathrm{~s}^{-1}$. Figure 12 shows the dependences of $d V_{t} / d Z$ on different factors in Eq. (14), where the same scale and slope parameters as shown in Fig. 11 are employed. As indicated, $d V_{t} / d Z$ almost bears no correlation to the factors $Z_{1} d V_{t 1}+Z_{2} d V_{t 2}$ and $\left(Z_{1} d V_{t 1}+Z_{2} d V_{t 2}\right) /\left(d Z_{1}+d Z_{2}\right)$. This implies that the contribution of $\Pi$ to $d V_{t} / d Z$ is very minor and can be neglected. However, from Fig. 12 a one-toone correspondence between the slope $d V_{t} / d Z$ and the factors $d \ln Z_{2}-d \ln Z_{1}$ and $\left(d \ln Z_{2}-d \ln Z_{1}\right) /\left(d Z_{1}+d Z_{2}\right)$ is clearly found, in which the sign of $d V_{t} / d Z$ is negative and that of $\Delta$ (or $d \ln Z_{2}-d \ln Z_{1}$ ) is positive (or negative). This result strongly suggests that the sign and magnitude of $\Delta$ both play crucial roles in dominating the behavior of $d V_{t} / d Z$.

Figure 13 shows an example of the dependence of $V_{t}-Z$ relation on the sign and magnitude of $\Delta$, in which a quasilinear relation between $V_{t}$ and $Z$ is seemingly demonstrated. It should be pointed out that the true $V_{t}-Z$ relation generally follows a power-law approximation, rather than linear relation (Chu et al., 1999; Protat et al., 2003; Su et al., 2004). The quasi-linear dependence of $V_{t}$ on $Z$ presented in Fig. 13 is the result of sufficiently small spans over the ranges of $V_{t}$ and $Z$. As shown, the slope of the quasi-linear $V_{t}-Z$ relation is not only a function of the magnitude of $\Delta$, but also determined by the sign of $\Delta$. It is clear from Fig. 13 that positive (negative) $\Delta$ corresponds to negative (positive) slope of the $V_{t}-Z$ relation, and the larger the absolute value of $\Delta$ is, the sharper the slope will be. The slope will approximate to infinity as the absolute value of $\Delta$ is close to infinity. Therefore, from expressions (14-16) and the results presented in Figs. 12 and 13, it is suggested that the condition $|\Delta| \rightarrow \infty$ serves as a threshold in the determination of the sign of $d V_{t} / d Z$ (or, equivalently, correlation between $V_{t}$ and $Z$ ) 


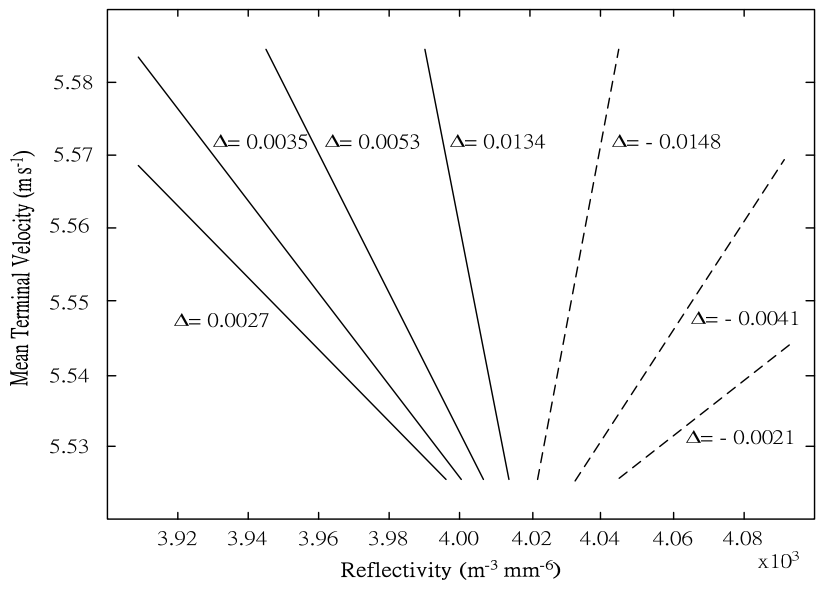

Fig. 13. Variation of the slope of $V_{t}-Z$ relation with magnitude of $\Delta$ that is defined by expression (15).

for the drop size distribution composed of two Gamma drop size distributions. However, it may not be true for the drop size distribution composed of three or more Gamma distributions. We will discuss these cases in another paper.

It is noteworthy that the same interaction process between precipitation particles might cause opposite $V_{t}-Z$ correlation, depending on the amount of the change in total radar reflectivity. For instance, for coalescence process, there is a tendency for the precipitation particles with larger size (or larger mean terminal velocity) to increase their number and those with smaller size to decrease. As a result, the radar refelctivities $Z_{1}$ and $Z_{2}$ are, respectively, increased and decreased accordingly, giving rise to $d \ln Z_{2}-d \ln Z_{1}<0$. In this case, the sign of $\Delta$ is dependent on the total change in radar reflectivity $d Z\left(=d Z_{1}+d Z_{2}\right)$. If the amount of the increase in $Z_{1}$ is smaller (or larger) that of the decrease in $Z_{2}$, i.e., $d Z_{1}+d Z_{2}<0$ (or $d Z_{1}+d Z_{2}>0$ ), the correlation between $V_{t}$ and $Z$ is then expected to be negative (or positive). With the similar argument, for the break-up process of the larger drops, the negative or positive $V_{t}-Z$ correlation would be obtained, depending on the sign of $d Z$. Table 2 summarizes the one-to-one correspondences between $V_{t}-Z$ correlations and the changes in the radar reflectivities in accordance with the analytical expressions (14-16), in which the plausible precipitation processes responsible for the reflectivity changes are also included for illustration. As shown in Table 2, there is no clear one-to-one correspondence between $V_{t}-Z$ correlation and the precipitation process that causes the change in the radar reflectivity. Therefore, it appears that the observed $V_{t}-P$ correlation made with a wind profiler would be very unlikely to be used to infer the physical process of the interaction of the precipitation particles.
Table 3. Analytical analysis of $V_{t}-Z$ correlation.

\begin{tabular}{ccccc}
\hline$V_{t}-Z$ correlation & $d Z_{1}$ & $d Z_{2}$ & $d Z$ & Plausible process \\
\hline negative & & & $>0$ & \\
positive & $<0$ & $>0$ & & Break-up \\
negative & & & $<0$ & \\
positive & $>0$ & $<0$ & & Coalescence \\
\hline
\end{tabular}

Note: $V_{t 1}>V_{t 2}$ is assumed.

\section{Conclusion}

With the help of the Chung-Li VHF radar, it is found that, irrespective of it prevails around the melting layer region, the negative correlation between reflectivity-weighted terminal velocity and echo power from precipitation can occur in the wide height regions extended from very much below to well above the melting layer for both convective and stratiform precipitations. This feature obviously cannot be explained in terms of the conventional $V_{t}-Z$ relations that were derived from single drop size distribution and predict positive correlation between $V_{t}$ and $Z$ for all precipitation environments. In order to explore the physics behind the negative correlation between $V_{t}$ and $Z$, a scenario that the precipitation particle size distribution for raindrop is composed of two different Gamma drop size distributions is proposed in this article, which have their own mean terminal velocities and radar reflectivities. It is assumed that the interaction of the precipitation particles between these two Gamma drop size distributions leads to the changes in the particle numbers (or, equivalently, radar reflectivities) of these two distributions. It is found from analytical analysis that the most crucial factor determining the occurrence of the negative correlation between $V_{t}$ and $Z$ is the ratio of the difference between the relative changes in the reflectivities to the total changes in the radar reflectivity. The $V_{t}-Z$ correlation will be positive (negative) if the ratio is negative (positive). From these arguments, it suggests that the negative $V_{t}-P$ correlation observed by VHF wind profiler can be theoretically attributed to the dynamic changes in the radar reflectivities of two different Gamma drop size distributions that constitute the observed drop size distribution. For the case that the net change in the total radar reflectivity is increased, the correlation between $V_{t}$ and $Z$ is expected to be positive (negative) when the difference between the fractional changes in the radar reflectivites of the two Gamma drop size distributions is negative (positive). On an analogous manner, for the case of the negative change in the total radar reflectivity, the $V_{t}-Z$ correlation is negative (positive) when the difference in the fractional changes in the radar reflectivites is negative 
(positive). Our theoretical analyses indicate that precipitation microphysical process such as coalescence (or break-up) can lead to both negative and positive $V_{t}-Z$ correlations. Thus, it appears that radar-observed $V_{t}-P$ correlations are unlikely to be used to infer the microphysical process in a precipitating cloud.

Acknowledgements. This work was partially supported by the National Science Council of the Republic of China in Taiwan under grant NSC 95-2111-M-008-015-MY3.

Topical Editor U.-P. Hoppe thanks E. Campos and another anonymous referee for their help in evaluating this paper.

\section{References}

Atlas, D., Srivastava, R. C., and Sekhon, R. S.: Doppler radar characteristics of precipitation at vertical incidence, Rev. Geophys. Space Phys., 11, 1-35, 1973.

Battan, L. J.: Radar observations of the atmosphere, pp. 324, University of Chicago Press, Chicago, III, 1973.

Beard, K. V.: Simple altitude adjustments to raindrop velocities for Doppler radar analysis, J. Atmos. Oceanic Technol., 2, 468-471, 1985.

Chilson, P. B., Ulbrich, C. W., Larsen, M. F., Perillat, P., and Keener, J. E.: Observations of a tropical thunderstorm using a vertically pointing, dual-frequency, collinear beam Doppler radar, J. Atmos. Oceanic Technol., 10, 663-673, 1993.

Chu, Y. H., Chian, L. P., and Liu, C. H.: The investigations of the atmospheric precipitation by using Chung-Li VHF radar, Radio Sci., 26, 717-729, 1991.

Chu, Y. H. and Lin, C. H.: Severe depletion of turbulent echo power in association with precipitation observed by using Chung-Li VHF Radar, Radio Sci., 29, 1311-1320, 1994.

Chu, Y. H. and Su, C. L.: Range resolution dependence of VHF radar returns from turbulence and precipitation, J. Atmos. SolarTerr. Phys., 61, 645-662, 1999.

Chu, Y. H., Shih, S. P., Su, C. L., Lee, K. L., Lin, T. H., and Liang, W. C.: A study on the relation between terminal velocity and VHF backscatter from precipitation particles using the Chung-Li VHF Radar, J. Appl. Meteor., 38, 1720-1728, 1999.
Chu, Y. H. and Ching-Lun, Su: An Investigation of Slope-Shape Relation for Gamma Raindrop Size Distribution, J. Appl. Meteor. Climatol., 47(10), 2531-2544, 2008.

Fukao, S., Wakasugi, K., Sato, T., Morimoto, S., Tsuda, T., Hirota, I., Kimura, I., and Kato, S.: Direct measurement of air and precipitation particle motion by very high frequency Doppler radar, Nature, 316, 712-714, 1985.

Foote, G. B. and du Toit, P. S.: Terminal velocity of raindrops aloft, J. Appl. Meteorol., 8, 585-591, 1969.

Houze Jr., R. A.: Cloud dynamics, Academic Press, 573 pp., 1993.

Kozu, T. and Nakamura, K.: Rainfall parameter estimation from dual-radar measurements combining reflectivity profiles and path-integrated attenuation, J. Atmos. Oceanic Technol., 8, 259270, 1991.

Larsen, M. F. and Rottger, J.: Observation of thunderstorm reflectivities and Doppler velocities measured at VHF and UHF, J. Atmos. Oceanic Technol., 4, 151-159, 1987.

Lhermitte, R. M. and Atlas, D.: Doppler fall speed and particle growth in stratiform precipitation; preprints of the 10th Radar Meteorology Conference, American Meteorological Society, 297-302, 1963.

Protat, A., Lemaître, Y., and Bouniol, D.: Terminal fall velocity and the FASTEX cyclones, Q. J. Roy. Meteor. Soc., 129(590), 15131535, 2003.

Sekhon, R. S. and Srivastava, R. C.: Doppler radar observations of drop-size distributions in a thunderstorm, J. Atmos. Sci., 28, 983-994, 1971.

Su, C. L., Chu, Y. H., and Chen, C. Y.: Analysis of Relation between Terminal Velocity of Rain Drop and VHF Backscatter from Precipitation, Terr. Atmos. Oceanic Sci., 15, 629-646, 2004.

Su, C. L. and Chu, Y. H.: Analysis of Terminal Velocity and VHF Backscatter of Precipitation Particles Using Chung-Li VHF Radar Combined with Ground-based Disdrometer, Terr. Atmos. Oceanic Sci., 18, 97-116, 2007.

Ulbrich, C. W.: Natural variation in the analytical form of the raindrop size distribution, J. Climate Appl. Meteor., 22, 1764-1775, 1983.

Ulbrich, C. W. and Chilson, P. B.: Effects of variations in precipitation size distribution and fallspeed law parameters on relations between mean Doppler fallspeed and reflectivity factor, J. Atmos. Oceanic Technol., 11, 1656-1663, 1994. 